\title{
Adrenal cortical carcinoma with tumor thrombus extension into the right atrium: A case report
}

\author{
YIMIN WANG ${ }^{*}$, FENG ZHOU* ${ }^{*}$, HAO PAN and BAIYE JIN \\ Department of Urology, The First Affiliated Hospital, College of Medicine, \\ Zhejiang University, Hangzhou, Zhejiang 310003, P.R. China
}

Received January 19, 2015; Accepted March 1, 2016

DOI: $10.3892 / \mathrm{ol} .2016 .4502$

\begin{abstract}
Adrenal cortical carcinoma (ACC) is a rare aggressive malignant tumor associated with a poor prognosis. Patients with ACC usually present with abnormal hormone secretion, tumor growth or encroachment on the surrounding viscera. An early diagnosis of ACC is considered challenging. The present study reports a case of ACC with tumor thrombus extension into the inferior vena cava and right atrium. A 33-year-old male patient exhibited symptoms of gynecomastia and abdominal distention, which were due to the abnormal hormone secretion levels and to the presence of a large mass in the right adrenal gland. An en bloc resection of the tumor was the selected treatment option. Extirpation of the tumor thrombus was performed by means of extracorporeal circulation. No signs of metastasis or recurrence were identified at the 1-month follow-up.
\end{abstract}

\section{Introduction}

Adrenal cortical carcinoma (ACC) is a rare malignant tumor known to have a poor prognosis. The incidence rate for ACC has been reported to be $\sim 0.5-2$ million individuals/year (1-3). It has a bimodal age distribution that peaks in children in the first decade of life and adults in the fourth to fifth decades of life, although the majority of patients with ACC are adults. ACC has a poor overall survival rate of $16-37 \%$ despite treatment (4). The majority of adrenal tumors are sporadic and unilateral, but 2-6\% are bilateral and are associated with Li-Fraumeni syndrome, multiple endocrine neoplasia type I, Beckwith-Wiedemann syndrome and the Carney complex, primarily in children (4). Tumor-associated symptoms of ACC

Correspondence to: Professor Baiye Jin, Department of Urology, The First Affiliated Hospital, College of Medicine, Zhejiang University, 79 Qingchun Road, Hangzhou, Zhejiang 310003, P.R. China

E-mail: zjuurology@163.com

${ }^{*}$ Contributed equally

Key words: adrenal cortical carcinoma, inferior vena cava, tumor thrombus, extracorporeal circulation, right atrium may be secondary to local or systemic disease burden and/or hypersecretion of adrenal hormones, which has been observed in $50-79 \%$ of adults and $90 \%$ of children with ACC (2). ACCs are classified into functional and non-functional tumors. It has been reported that $1 / 3$ of patients with ACC also present with tumor thrombi that extend into the inferior vena cava (IVC), $51 \%$ of which also extend into the right atrium. The standard treatment for ACC is complete surgical removal of the primary tumor, which may require the resection of adjacent organs, such as the kidneys and spleen, and any caval thrombus that are present, as well as en bloc resection of local lymph nodes (5). Mitotane has been administered for patients with metastatic disease, however, despite the response rates exhibited, no improvement in survival time was identified following treatment $(6,7)$. The present study reports a case of functional $\mathrm{ACC}$ in the right adrenal, inferior to the right lobe of the liver with tumor thrombus extension into the IVC and right atrium.

\section{Case report}

A 33-year-old male patient was admitted to The First Affiliated Hospital, College of Medicine of Zhejiang University (Hangzhou, China) on May 13, 2013 presenting with symptoms of gynecomastia for 3 months and abdominal distention for 1 month. Abdominal computed tomography angiography (Brilliance iCT; Philips Healthcare, DA Best, The Netherlands) revealed a large mass $18 \times 15 \mathrm{~cm}$ in size in the right adrenal gland, located below the right lobe of the liver, with tumor thrombus extension into the IVC. The left renal vein had been pushed down by the mass (Fig. 1). Magnetic resonance imaging scan (Signa HDx 3.0T; GE Healthcare Life Sciences, Chalfont, UK) of the IVC revealed a large mass in the right adrenal gland with tumor thrombus extension into both the IVC and the right atrium (Fig. 2). Echocardiography (IE33; Philips Healthcare) also identified the tumor thrombus in the right atrium (Fig. 3). Chest X-ray showed no metastasis.

The hormone levels of the patient were also measured. The serum aldosterone values were as follows: Upright position, $634.02 \mathrm{ng} / \mathrm{dl}$ (normal values, 70.00-300.00 ng/dl) and supine position, $434.90 \mathrm{ng} / \mathrm{dl}$ (normal values, 30.00-160.00 ng/dl). Angiotensin II values were as follows: Upright position, 86.10 (normal values, 50.00-120.00) and supine position, 85.62 (normal values, 25.00-60.00). Plasma renin activity (PRA) values were as follows: Upright position, 15.36 (normal values, 


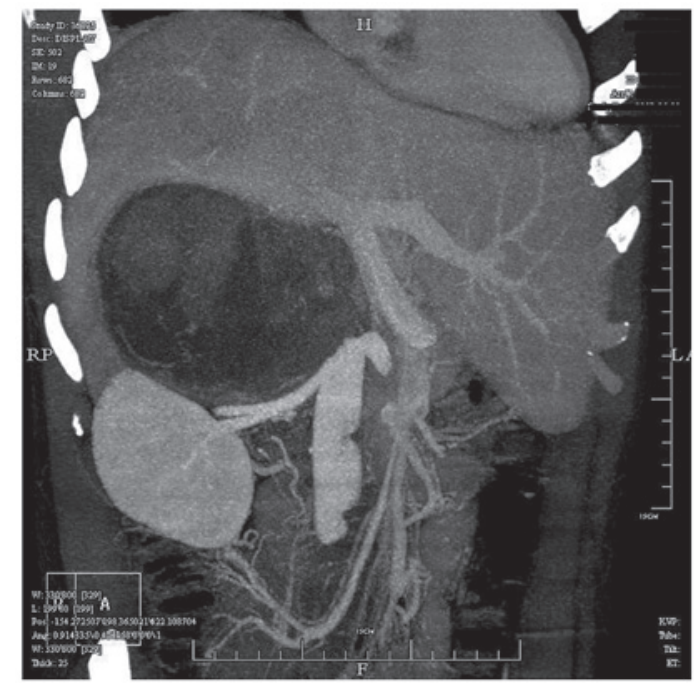

Figure 1. Computed tomography angiography revealed a large mass $13.5 \times 9.5 \mathrm{~cm}$ in size in the right adrenal gland, located below the right lobe of the liver, with tumor thrombus extension into the inferior vena cava.

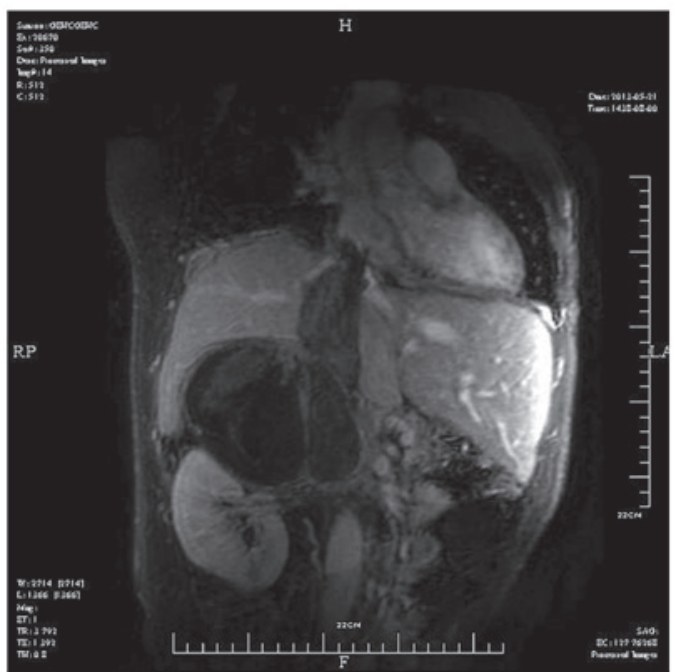

Figure 2. Magnetic resonance imaging scan of the IVC revealed a large mass $13.5 \times 9.5 \mathrm{~cm}$ in size located in the right adrenal gland, with tumor thrombus extension into both the IVC and right atrium. IVC, inferior vena cava.

0.10-6.56) and supine position, 0.91 (normal values, $0.15-2.33$ ). Testosterone levels were $26.20 \mathrm{ng} / \mathrm{dl}$ (normal values in men, $262.00-1,593.00 \mathrm{ng} / \mathrm{dl})$. Estradiol levels were $208.00 \mathrm{pg} / \mathrm{ml}$ (normal values, $0.00-56.00 \mathrm{pg} / \mathrm{ml}$ ). The levels of follicle stimulating hormone $(\mathrm{FSH})$ were $0.20 \mathrm{mIU} / \mathrm{ml}$ (normal values, $0.70-11.10 \mathrm{mIU} / \mathrm{ml}$ ), while the levels of luteinizing hormone (LH) were $1.40 \mathrm{mIU} / \mathrm{ml}$ (normal values, $0.80-7.60 \mathrm{mIU} / \mathrm{ml}$ ). The levels of prolactin were $19.30 \mathrm{ng} / \mathrm{ml}$ (normal values, 2.50-17.00 ng/ml), while the levels of progesterone were $1.18 \mathrm{ng} / \mathrm{ml}$ (normal values, $0.27-0.90 \mathrm{ng} / \mathrm{ml}$ ). The levels of all other adrenalcortical hormones were normal.

The patient underwent a thoracoabdominal incision to achieve the best exposure of the tumor. Radical right nephrectomy and adrenalectomy were performed. The tumor was observed to be located very close to the adjacent viscera, particularly to the right kidney, IVC and right lobe of the liver, and the period of time required for separating

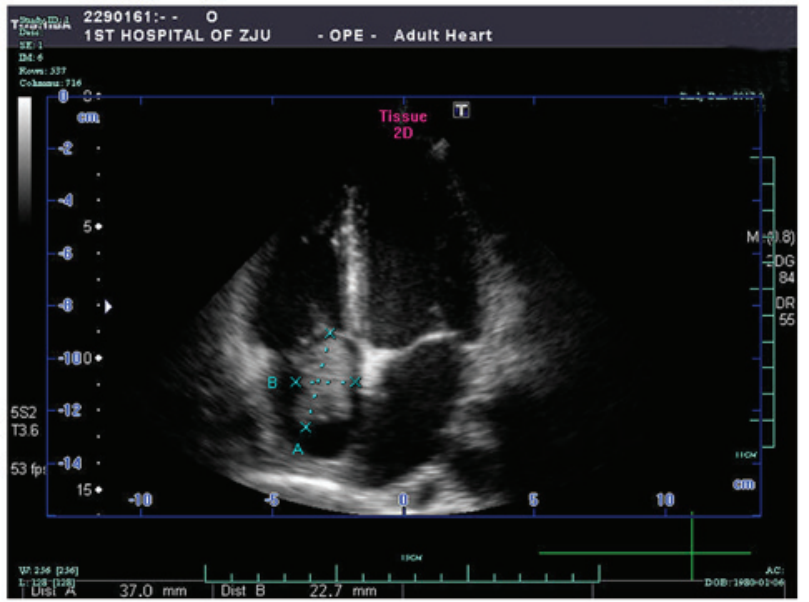

Figure 3. Echocardiography revealed the presence of the tumor thrombus in the right atrium.

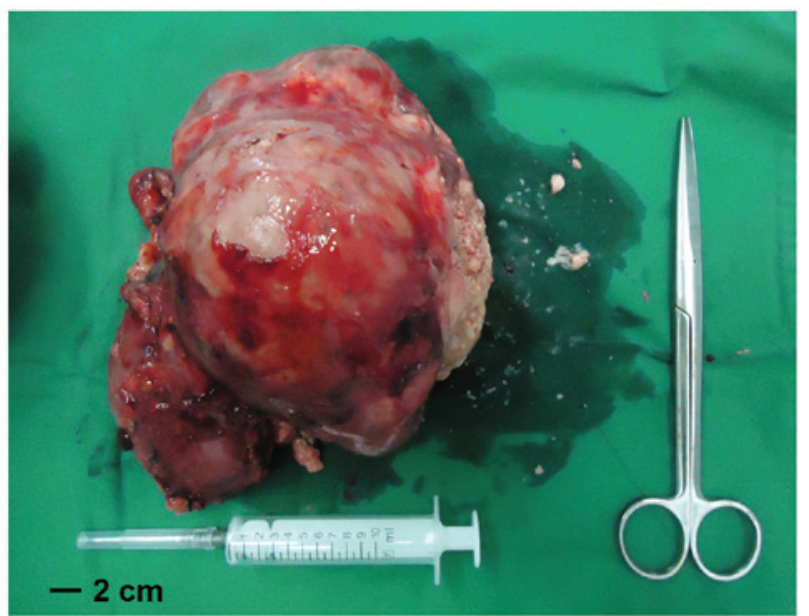

Figure 4. Image of the external surface of the tumor, which measured $\sim 18 \mathrm{x} 15 \mathrm{~cm}$ and weighted $1.5 \mathrm{~kg}$, including the weight of the right kidney.

the tumor from the organs was considerably lengthy. The right kidney was enveloped by the tumor, and thus had to be removed; however, the right lobe of the liver was successfully separated from the tumor. The tumor measured $\sim 18 \times 15 \mathrm{~cm}$ and weighted $1.5 \mathrm{~kg}$, including the weight of the right kidney (Fig. 4). Following consultation with hepatobiliary and pancreatic, the patient was subjected to vascular surgery. A resection of the right lobe of the liver and IVC replacement were considered. However, the surgeons (Department of Urology, The First Affiliated Hospital, Zhejiang University) managed to separate the tumor from the viscera, thus avoiding both of these procedures, although the estimated blood loss was $\sim 3,000 \mathrm{ml}$.

In order to extract the tumor thrombus, which had extended into the IVC and right atrium, the right lobe of the liver had to be mobilized. Therefore, extracorporeal circulation (8) was performed by cardiothoracic surgeons (Department of Urology, The First Affiliated Hospital, Zhejiang University). The superior and inferior venae cavae, first hepatic portal artery and vein, and left renal vein were clamped, in order to reduce bleeding. Subsequently, the right atrium was opened, 
and the tumor thrombus was successfully removed from the IVC and right atrium under visual control, using extracorporeal circulation assisted by cardiothoracic surgeons. The tumor thrombus exhibited a gelatinous and friable appearance (Fig. 5 and 6). The portal and renal vein were repeatedly opened and closed, in order to irrigate the visible tumor thrombus and prevent residual tumor thrombus and embolism. A segment wall of the IVC was excised due to tumor thrombus invasion, and repaired using the pericardial patch repair technique (9). The procedure was uneventful. The patient returned to the ward after 1 day in the intensive care unit. The patient was administered adjuvant treatment with $4 \mathrm{~g}$ mitotane daily 7 days following surgery, and was discharged 12 days following surgery. No signs of metastasis or recurrence were identified at the 1-month follow-up.

The hormone levels of the patients were measured again 7 days following surgery. The serum aldosterone levels were as follows: Upright position, $327.11 \mathrm{ng} / \mathrm{dl}$ (normal values, 70.00-300.00 ng/dl) and supine position, $184.67 \mathrm{ng} / \mathrm{dl}$ (normal values, 30.00-160.00 ng/dl). The angiotensin II levels were as follows: Upright position, $85.71 \mathrm{ng} / 1$ (normal values, 50.00-120.00 ng/l) and supine position, $77.84 \mathrm{ng} / 1$ (normal values, $25.00-60.00 \mathrm{ng} / \mathrm{l})$. PRA values were as follows: Upright position $>24.00 \mathrm{ng} / \mathrm{ml} / \mathrm{h}$ (normal values, $0.10-6.56 \mathrm{ng} / \mathrm{ml} / \mathrm{h}$ ) and supine position, $5.16 \mathrm{ng} / \mathrm{ml} / \mathrm{h}$ (normal values, $0.15-2.33 \mathrm{ng} / \mathrm{ml} / \mathrm{h}$ ). Testosterone levels were at $138.00 \mathrm{ng} / \mathrm{dl}$ (normal values in men, 262.00-1,593.00 ng/dl). Estradiol levels were $101.00 \mathrm{pg} / \mathrm{ml}$ (normal values, 0.00-56.00 pg/ml). The levels of FSH were $2.70 \mathrm{mIU} / \mathrm{ml}$ (normal values, $0.70-11.10 \mathrm{mIU} / \mathrm{ml}$ ) and those of $\mathrm{LH}$ were $11.20 \mathrm{mIU} / \mathrm{ml}(0.80-7.60 \mathrm{mIU} / \mathrm{ml})$. The levels of prolactin were $18.60 \mathrm{ng} / \mathrm{ml}$ (normal values, $2.50-17.00 \mathrm{ng} / \mathrm{ml}$ ), and those of progesterone were $1.32 \mathrm{ng} / \mathrm{ml}$ (normal values, $0.27-0.90 \mathrm{ng} / \mathrm{ml}$ ). All other adrenal cortical hormone levels were normal. The post-surgical levels of aldosterone had decreased compared with the preoperative levels, although they remained considerably high. PRA also remained at a high level.

Resected specimens were formalin (Beijing Solarbio Science \& Technology Co., Ltd., Beijing, China)-fixed, paraffin (Beijing Solarbio Science \& Technology Co., Ltd.)-embedded and cut into 3- $\mu \mathrm{m}$ sections. Histopathological staining with hematoxyli $\mathrm{n}$ and eosin (Beijing Solarbio Science \& Technology Co., Ltd.) confirmed the diagnosis of adrenal cortical carcinoma with associated tumor thrombus. Tissue sections were incubated with primary antibodies overnight at $4^{\circ} \mathrm{C}$. Immunohistochemical findings included the following (Fig. 7): Pan-cytokeratin ${ }^{+}$(mouse monoclonal; catalog no., Kit-0009), synuclein ${ }^{+}$(mouse monoclonal; catalog no., MAB-0538), S-100- (mouse monoclonal; catalog no., Kit-0007), melan- $\mathrm{A}^{+}$(mouse monoclonal; catalog no., MAB-0275), hepatocyte marker (mouse monoclonal; catalog no., MAB-0249) and 70\% Ki-67+ (mouse monoclonal; catalog no., Kit-0005) (Fuzhou Maixin Biotech Co., Ltd.).

The patient succumbed to the disease 18 months subsequent to surgery due to extensive metastasis. Written informed consent was obtained from the patient for the publication of the present study.

\section{Discussion}

ACC is a rare type of malignant tumor associated with a poor prognosis (1-3). The incidence rate for ACC has been reported

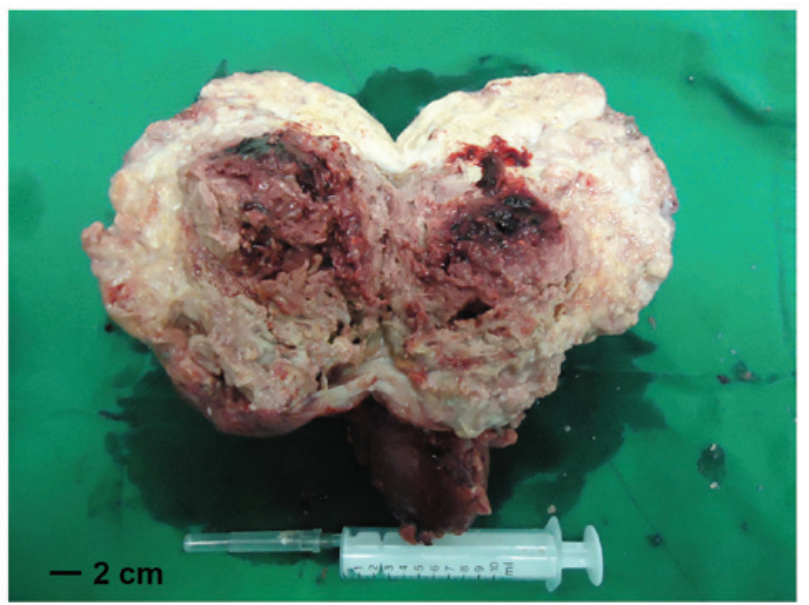

Figure 5. Image of the internal structure of the tumor. Hemorrhage and necrosis was observed on cross-section.

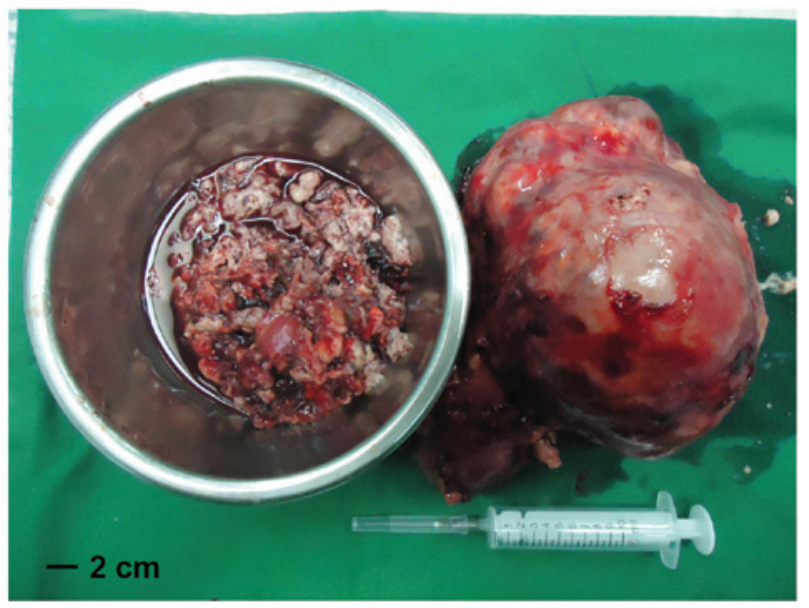

Figure 6. The tumor thrombus exhibited a gelatinous and friable appearance.

to be $\sim 0.5-2 / 1,000,000 /$ year $(10,11)$. ACC can be classified into functional and non-functional, based on hormone levels (functional, abnormal hormone secretion; non-functional, normal hormone secretion). Hypercortisolism is considered to be the most common symptom of functional ACC, while primary hyperaldosteronism secondary to ACC with symptoms of hypertension and hypokalemia is rare $(2,12)$. ACC with abnormal levels of reproductive hormones is also rare (2). Androgen-secreting ACCs can induce hirsutism and virilization in women, while estrogen-secreting adrenal tumors can cause gynecomastia and testicular atrophy in men (13-15). Tritos et al (16) reported that ACC functional tumors are associated with worse survival than non-functional tumors. By contrast, other studies did not identify any association between tumor status and survival in patients with ACC $(7,17)$. In the present case, the tumor caused the patient's endocrine system to malfunction. Due to its irregularity, the endocrine status of a patient is may be difficult to explain (2). In the present patient, the aldosterone and estradiol levels increased, while the testosterone levels decreased, which resulted in the presentation of gynecomastia. The patient complained of bilateral breast pain and swelling, without testicular atrophy or impotence. In 

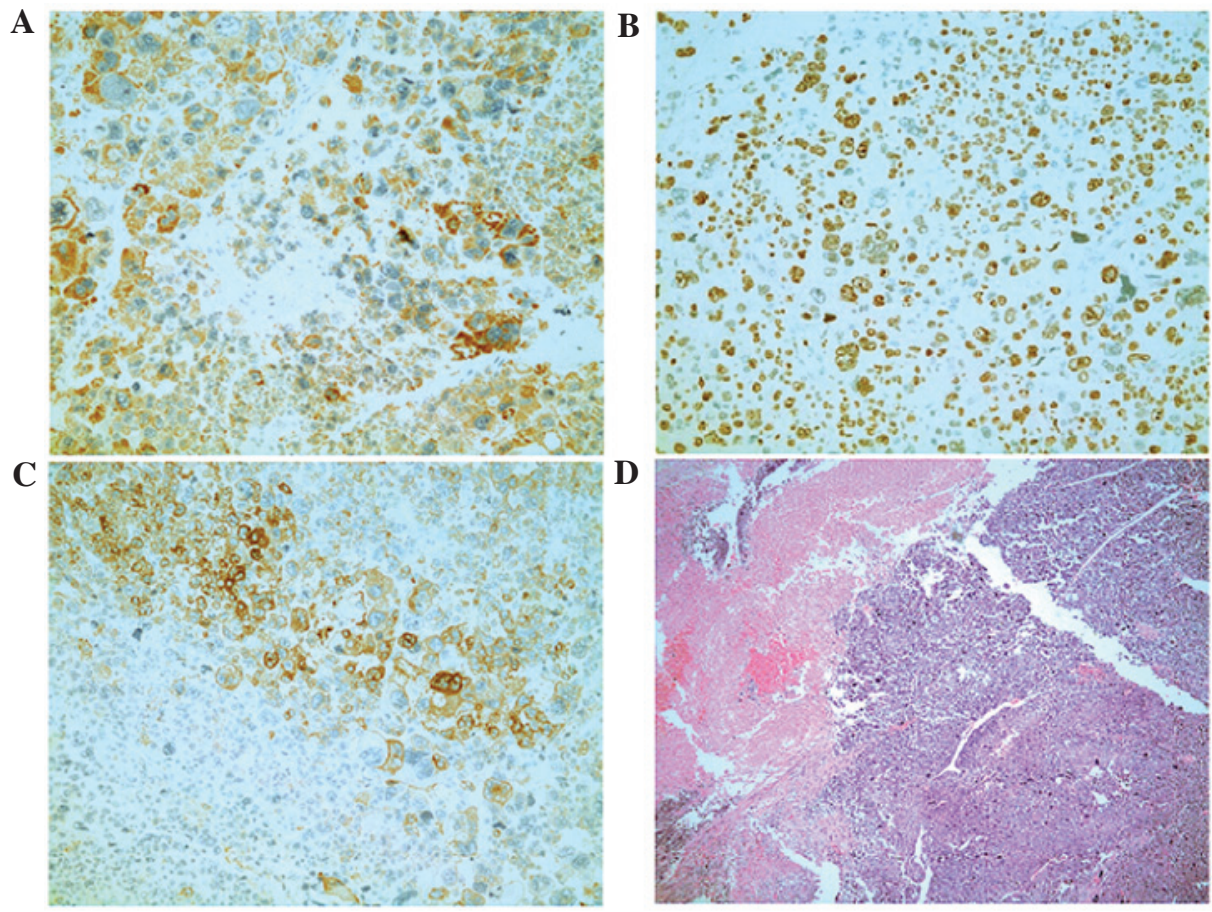

Figure 7. Immunohistochemical staining for (A) melan-A, (B) Ki-67 and (C) synuclein. (D) Histopathological confirmation of the diagnosis of adrenal cortical carcinoma with associated tumor thrombus (stain, hematoxylin and eosin). Magnification, x50.

addition, the patient presented with abdominal distention, which was due to the large size of the tumor.

ACC is an aggressive tumor, whose early symptoms are not obvious and its prognosis is poor $(2,9)$. The median survival for untreated patients is 2.5 months (18). Based on the staging system established by the European Network for the Study of Adrenal Tumors (ENS@T) (19), the present patient was diagnosed with stage III ACC. Tritos et al (16) analyzed 31 cases of ACC and observed that the prognostic factors were associated with the time of diagnosis, age, absence of metastatic disease, tumor stage and whether or not complete surgical resection had been performed. By contrast, the authors did not identify any association between prognosis and gender or tumor thrombus extension into the IVC. The median survival of patients with ACC may be prolonged to 17 months, or even 26 months if the patient has undergone radical surgery (9). There have been numerous reports of cases where radical resection of the tumor was independently associated with improved survival $(17,20,21)$.

ACC with tumor thrombus extension into the IVC is considered a rare occurrence (18). The majority of patients with this condition present with large tumors, and $51 \%$ of these tumors extend into the right atrium (18). ACC with tumor thrombus extension into the right atrium alone should not be considered a contraindication against radical surgical therapy (15). Despite the increased risk associated with the surgical procedure, clinicians should attempt a radical resection or a maximal tumor load debulking (15). In general, extracting the tumor thrombus under extracorporeal circulation is relatively safe, if the patient has no other systemic disorders $(8,9)$. This method may reduce the duration of the surgery, minimize the blood loss and prevent avoid embolism (9). Preoperative planning and intraoperative cooperation among multiple departments are imperative for this type of surgical procedure. In the present case, the tumor was removed en bloc, and the tumor thrombus was extracted under extracorporeal circulation. The procedure was successful and uneventful. According to Chiche et al (15), only in 3/15 cases were vena cava resection or graft replacement required, whereas opposite side renal vein resection and graft replacement was not required in any of the cases included in that study. In the present case, if the separation of the tumor from the viscera had been rendered impossible, resection of the right lobe of the liver or graft replacement of the IVC would have been required. In the end, the tumor was successfully separated from the viscera and the patient recovered well from the surgery.

The patient was administered adjuvant treatment 7 days following surgery, which consisted of $4 \mathrm{~g}$ mitotane daily, in order to maintain plasma concentrations of the drug of $14-20 \mathrm{mg} / 1$ (22). Adjuvant mitotane therapy for ACC is controversial, since there is a limited number of randomized controlled trials, due to the low incidence of the disease. Early studies disagreed on whether mitotane was able to improve patient survival $(7,17,23)$. A subsequent retrospective study (24) demonstrated that mitotane can improve recurrence-free survival but not overall survival in patients with ACC. In 2010, a panel of international experts proposed adjuvant mitotane treatment for ACC patients with potential residual disease or $>10 \%$ Ki67 positivity detected upon immunohistochemical examination (25). The present patient was administered mitotane, since, according to the ENS@T staging system, the patient exhibited stage III disease and $70 \%$ Ki67 positivity. No signs of metastasis or recurrence were identified at the 1-month follow-up.

Based on the available literature, as well as personal experience, the present authors propose that extension of 
the tumor thrombus into the right atrium alone should not be considered a contraindication against radical surgical therapy in patients with ACC. However, preoperative planning and intraoperative cooperation among multiple departments for the surgical procedure are imperative. In conclusion, despite the association of ACC with poor prognosis, radical surgery-based therapy may result in favorable outcomes for ACC patients.

\section{References}

1. Aubert S, Wacrenier A, Leroy X, Devos P, Carnaille B, Proye C, Wemeau JL, Lecomte-Houcke M and Leteurtre E: Weiss system revisited: A clinicopathologic and immunohistochemical study of 49 adrenocortical tumors. Am J Surg Pathol 26: 1612-1619, 2002.

2. Roman S: Adrenocortical carcinoma. Curr Opin Oncol 18: 36-42, 2006

3. Fassnacht M and Allolio B: Clinical management of adrenocortical carcinoma. Best Pract Res Clin Endocrinol Metab 23: 273-289, 2009.

4. Ng L and Libertino JM: Adrenocortical carcinoma: Diagnosis, evaluation and treatment. J Urol 169: 5-11, 2003.

5. Ekici S and Ciancio G: Surgical management of large adrenal masses with or without thrombus extending into the inferior vena cava. J Urol 172: 2340-2343, 2004.

6. Hoffman DL and Mattox VR: Treatment of adrenal cortical carcinoma with o,p'-DDD. Med Clin North Am 56: 999-1012, 1972.

7. Luton JP, Cerdas S, Billaud L, Thomas G, Guilhaume B, Bertagna X, Laudat MH, Louvel A, Chapuis Y and Blondeau P: Clinical features of adrenocortical carcinoma, prognostic factors, and the effect of mitotane therapy. N Engl J Med 322: 1195-1201, 1990

8. Nardi P, Pellegrino A, Pugliese M, Bovio E, Chiariello L and Ruvolo G: Cardiac surgery with extracorporeal circulation and concomitant malignancy: Early and long-term results. J Cardiovasc Med (Hagerstown) 17: 152-159, 2016.

9. Bertherat J, Coste J and Bertagna X: Adjuvant mitotane in adrenocortical carcinoma. N Engl J Med 357: 1256-1257, 2007.

10. Kerkhofs TM, Verhoeven RH, Van der Zwan JM, Dieleman J, Kerstens MN, Links TP, Van de Poll-Franse LV and Haak HR: Adrenocortical carcinoma: A population-based study on incidence and survival in the Netherlands since 1993. Eur J Cancer 49: 2579-2586, 2013.

11. Kebebew E, Reiff E, Duh QY, Clark OH and McMillan A: Extent of disease at presentation and outcome for adrenocortical carcinoma: Have we made progress? World J Surg 30: 872-878, 2006.
12. Puvaneswary $\mathbf{M}$ and Thong $\mathrm{K}$ : Primary hyperaldosteronism: Due to adrenal cortical carcinoma. Australas Radiol 37: 88-89, 1993.

13. Coonrod DV and Rizkallah TH: Virilizing adrenal carcinoma in a woman of reproductive age: A case presentation and literature review. Am J Obstet Gynecol 172: 1912-1915, 1995.

14. Lanigan D, Choa RG and Evans J: A feminizing adrenocortical carcinoma presenting with gynaecomastia. Postgrad Med J 69: 481-483, 1993.

15. Chiche L, Dousset B, Kieffer E and Chapuis Y: Adrenocortical carcinoma extending into the inferior vena cava: Presentation of a 15-patient series and review of the literature. Surgery 139: 15-27, 2006.

16. Tritos NA, Cushing GW, Heatley G and Libertino JA: Clinical features and prognostic factors associated with adrenocortical carcinoma: Lahey Clinic Medical Center experience. Am Surg 66: 73-79, 2000.

17. Bodie B, Novick AC, Pontes JE, Straffon RA, Montie JE, Babiak T, Sheeler L and Schumacher P: The Cleveland Clinic experience with adrenal cortical carcinoma. J Urol 141: 257-260, 1989.

18. Hedican SP and Marshall FF: Adrenocortical carcinoma with intracaval extension. J Urol 158: 2056-2061, 1997.

19. Lebastchi AH, Kunstman JW and Carling T: Adrenocortical carcinoma: Current therapeutic state-of-the-art. J Oncol 2012: 234726, 2012.

20. Allolio B, Hahner S, Weismann D and Fassnacht M: Management of adrenocortical carcinoma. Clin Endocrinol (Oxf) 60: 273-287, 2004.

21. Dackiw AP, Lee JE, Gagel RF and Evans DB: Adrenal cortical carcinoma. World J Surg 25: 914-926, 2001.

22. Ochi T, Tanji N, Shimamoto K, Ikeda T, Toshino A and Yokoyama M: Application of cardiopulmonary bypass for resection of renal cell carcinoma and adrenocortical carcinoma extending into the right atrium. Int J Urol 13: 202-205, 2006.

23. Haak HR, Hermans J, van de Velde CJ, Lentjes EG, Goslings BM, Fleuren GJ and Krans HM: Optimal treatment of adrenocortical carcinoma with mitotane: Results in a consecutive series of 96 patients. Br J Cancer 69: 947-951, 1994.

24. Terzolo M, Angeli A, Fassnacht M, Daffara F, Tauchmanova L, Conton PA, Rossetto R, Buci L, Sperone P, Grossrubatscher E, et al: Adjuvant mitotane treatment for adrenocortical carcinoma. N Engl J Med 356: 2372-2380, 2007.

25. Berruti A, Fassnacht M, Baudin E, Hammer G, Haak H, Leboulleux S, Skogseid B, Allolio B and Terzolo M: Adjuvant therapy in patients with adrenocortical carcinoma: A position of an international panel. J Clin Oncol 28: e401-e402, 2010. 